\title{
ON MYCIELSKI IDEALS
}

\author{
MAREK BALCERZAK AND ANDRZEJ ROSLANOWSKI
}

(Communicated by Andreas R. Blass)

\begin{abstract}
We investigate relationships between Mycielski ideals in $2^{\omega}$ generated by different systems. For a fixed Mycielski ideal $\mathfrak{M}$ we study properties of its compact members. For a perfect Polish space $X$ and certain sets $A \subseteq X \times 2^{\omega}$, the positions of $\left\{x \in X: A_{X} \notin \mathfrak{M}\right\}$ in the Borel and projective hierarchies are established and other section properties are observed.
\end{abstract}

\section{INTRODUCTION}

The notation concerning descriptive set theory will be derived from [Mo] and the set-theoretical notation will be standard. For example, for any finite sequence $s \in 2^{<\omega}$, let $[s]$ denote the clopen basic set in $2^{\omega}$ generated by $s$. For $A \subseteq X \times 2^{\omega}$ and $x \in X$, let $A_{x}=\left\{c \in 2^{\omega}:(x, c) \in A\right\}$. Similarly $A^{d}$ for $d \in 2^{\omega}$. A pointclass $\mathscr{R}$ is called closed under continuous substitution if, for any perfect Polish spaces $X, Y$ and a continuous function $f: X \rightarrow Y$, we have $f^{-1}[E] \in \mathscr{R}(X)$ for all $E \in \mathscr{R}(Y)$. We shall use the symbols $\exists^{Y}, \forall^{Y}$, $\wedge^{\omega}$ defined as in [Mo, pp. 25-27 and 44].

Let $K \subseteq \omega$ be an infinite set for which $\omega \backslash K$ is infinite; such a $K$ will be called normal here. For $A \subseteq 2^{\omega}$, consider the game $\Gamma(A, K)$ with perfect information between two players, I and II. Player I chooses $c_{i} \in 2=\{0,1\}$ if $i \in \omega \backslash K$ and player II chooses $c_{i}$ if $i \in K$. Player I wins if $c=\left(c_{0}, c_{1}, \ldots\right) \in$ $A$ and player II wins in the opposite case (cf. [My]). A strategy is a function $\sigma: 2^{<\omega} \rightarrow 2$. Let STR denote the set of all strategies. This set with the obvious product topology is homeomorphic to the Cantor space $2^{\omega}$. For $\sigma, \tau \in$ STR and a normal $K$, we define $\sigma{ }^{*} \tau=c \in 2^{\omega}$ as follows

$$
c_{n}=\left\{\begin{array}{ll}
\sigma(c \mid n) & \text { if } n \notin K \\
\tau(c \mid n) & \text { if } n \in K
\end{array} \text { for } n \in \omega .\right.
$$

If we replace $\tau(c \mid n)$ by $d(n)$ (where $d \in 2^{\omega}$ ) in the above formula, then $\sigma *_{K} d$ will be defined. Similarly for $d *_{K} \tau$. The functions described above by ${ }_{K}{ }_{K}$ are continuous.

A strategy $\sigma$ is called winning for player I if $(\forall \tau \in \mathrm{STR})\left(\sigma{ }^{*}{ }_{K} \tau \in A\right)$. Similarly for player II. If one of the players has a winning strategy, the game

Received by the editors December 5, 1988 and, in revised form, May 23, 1989.

1980 Mathematics Subject Classification (1985 Revision). Primary 04A15; Secondary 90D05. 
$\Gamma(A, K)$ is called determined. Denote by $V_{\mathrm{II}}(K)$ the family of all sets $A \subseteq 2^{\omega}$ for which player II has a winning strategy in $\Gamma(A, K)$.

Assume that we have a fixed system $\left\{K_{s}\right\}_{s \in 2^{<\omega}}$ of normal sets such that $K_{s \hat{0}} \cap K_{s_{\hat{1} 1}}=\varnothing$ and $K_{s \hat{0}} \cup K_{s_{1} 1} \subseteq K_{s}$ for all $s \in 2^{<\omega}$ (where $\hat{s} i$ denotes the respective extension of $s)$. Let $\mathfrak{M}=\bigcap\left(V_{\mathrm{II}}\left(K_{s}\right): s \in 2^{<\omega}\right\}$. This is a $\sigma$-ideal introduced by Mycielski in [My]. It fulfills natural invariance conditions. Similar $\sigma$-ideals can be constructed if the players choose elements of an arbitrary set $X$, not necessarily $\{0,1\}$ (cf. [R]). Most of our results can be extended to this case.

In the sequel, $K$ will denote a normal set.

0.1. Lemma. There is a continuous function $f: 2^{\omega} \rightarrow 2^{\omega}$ such that $f^{-1}[\{d\}]$ $\notin V_{\mathrm{II}}(K)$ for all $d \in 2^{\omega}$.

Proof. Let $\omega \backslash K=\left\{n_{0}, n_{1}, \ldots\right\}$ and put $f(c)=\left(c_{n_{0}}, c_{n_{1}}, \ldots\right)$ for $c=\left(c_{0}\right.$, $\left.c_{1}, \ldots\right) \in 2^{\omega}$. In each game $\Gamma\left(f^{-1}[\{d\}], K\right)$ player I has a winning strategy, thus we get the assertion.

0.2. Lemma (cf. [My], Theorem 9). If $A \notin V_{\mathrm{II}}(K)$ and the game $\Gamma(A, K)$ is determined, there exists a closed $F \subseteq A$ such that $F \notin V_{\mathrm{II}}(K)$.

\section{DePENDENCE ON GENERATING SySTEMS}

It is remarked in $[R]$ that the ideal $\mathfrak{M}$ heavily depends on the system $\left\{K_{s}\right\}$. In this section we continue these considerations.

Let us recall some definitions. The pair $\mathfrak{J}, \mathfrak{F}$ of ideals in $\mathscr{P}\left(2^{\omega}\right)$ is called orthogonal if there are two sets $A \in \mathfrak{J}$ and $B \in \mathfrak{F}$ such that $A \cup B=2^{\omega}$. We say that a $\sigma$-ideal $\mathfrak{J}$ is sharper than a $\sigma$-ideal $\mathfrak{F}$ if there are $\sigma$-ideals $\mathfrak{J}^{*}, \mathfrak{F}^{*}$ such that both pairs $\mathfrak{J}, \mathfrak{J}^{*}$ and $\mathfrak{F}, \mathfrak{F}^{*}$ are orthogonal and $\mathfrak{J} \subseteq \mathfrak{F}^{*} \cap \mathfrak{F}$ (cf. $\left.\left[\mathrm{Ba}_{1}\right]\right)$.

The following question is formulated in $\left[\mathrm{Ba}_{1}\right]$. Does there exist an infinite sequence $\left(\mathfrak{J}_{n}\right\}$ of translation-invariant symmetric $\sigma$-ideals with Borel bases, such that $\mathfrak{J}_{n+1}$ is sharper than $\mathfrak{J}_{n}$ ?

We give an example of such a sequence of size $\omega_{1}$.

1.1. Lemma. For each countable family $\left\{\mathfrak{M}_{n}: n \in \omega\right\}$ of Mycielski ideals, there exists a Mycielski ideal $\mathfrak{M}$ which is orthogonal to every $\mathfrak{M}_{n}$.

Proof. Let $\left\{K_{s}^{n}\right\}_{s \in 2^{<\omega}}$ be the system generating $\mathfrak{M}_{n}$.

Claim. For each $L \subseteq \omega$ such that $L \cap K_{s}^{n} \neq \varnothing$ for all $s$ and $n$, there are disjoint sets $L_{0}, L_{1} \subseteq L$ with the same property.

Observe that the sets $L \cap K_{s}^{n}$ are in fact infinite, so the claim is clear.

Claim. There exists a system $\left\{K_{t}\right\}_{t \in 2}<\omega$ such that $K_{t} \cap K_{s}^{n} \neq \varnothing$ for all $s, t$ and $n$.

This is a consequence of the previous claim. 
Let $\mathfrak{M}$ be the ideal generated by the system $\left\{K_{t}\right\}$. Then $\mathfrak{M}$ is orthogonal to all $\mathfrak{M}_{n}$ 's (the set $A=\left\{c \in 2^{\omega}:\left(\forall t \in 2^{<\omega}\right)\left(\exists i \in K_{t}\right)(c(i)=0)\right\}$ belongs to $\mathfrak{M}$, while its complement belongs to all ideals $\mathfrak{M}_{n}$ ).

1.2. Lemma. For each countable family $\left\{\mathfrak{M}_{n}: n \in \omega\right\}$ of Mycielski ideals, there exists a Mycielski ideal $\mathfrak{M}$ contained in the intersection of $\mathfrak{M}_{n}$ 's.

Proof. Let $\left\{K_{s}^{n}\right\}_{s \in 2^{<\omega}}$ be the system generating $\mathfrak{M}_{n}$. Observe that there exists a system $\left\{K_{t}\right\}_{t \in 2^{<\omega}}$ such that $(\forall n)(\forall s)(\exists t)\left(K_{t} \subseteq K_{s}^{n}\right)$. Let $\mathfrak{M}$ be the ideal generated by the system $\left\{K_{t}\right\}$. Then $\mathfrak{M}$ is included in all $\mathfrak{M}_{n}$ 's.

1.3. Theorem. There exists a sequence $\left\{\mathfrak{M}_{\alpha}: \alpha<\omega_{1}\right\}$ of Mycielski ideals such that $\alpha<\beta$ that $\mathfrak{M}_{\beta}$ is sharper than $\mathfrak{M}_{\alpha}$.

Proof. We use induction and the previous lemmas.

Now, we show another fact illustrating the dependence of Mycielski ideals on generating systems. Mycielski in [My] proved that all ideals $\mathfrak{M}$ are orthogonal to the ideal $\mathbf{K}$ of meager sets in $2^{\omega}$. However, we have

1.4. Theorem. For each $A \in \mathbf{K}$, there exists a Mycielski ideal $\mathfrak{M}$ such that $A \in \mathfrak{M}$.

Proof. Let $G_{n}$ be dense open subsets of $2^{\omega}$ such that $G_{n+1} \subseteq G_{n}$ and

$$
\bigcap\left\{G_{n}: n \in \omega\right\} \cap A=\varnothing .
$$

Construct an increasing sequence $\left\{t_{n}: n \in \omega\right\} \subseteq \omega$ with the following property

$$
(\forall n)\left(\forall s: t_{n} \rightarrow 2\right)\left(\exists s^{\prime}: t_{n+1} \backslash t_{n} \rightarrow 2\right)\left(\left[s^{\wedge} s^{\prime}\right] \subseteq G_{n}\right) .
$$

Let $\left\{K_{s}\right\}$ be an arbitrary system. Put $K_{s}^{\prime}=\bigcup\left\{t_{n+1} \backslash t_{n}: n \in K_{s}\right\}$. Then $\left\{K_{s}^{\prime}\right\}$ generates the respective ideal

Remarks. (a) The above theorem shows that there is no Borel function $f: 2^{\omega} \rightarrow$ $2^{\omega}$ such that $f^{-1}[\{d\}] \notin \mathfrak{M}$ for all Mycielski ideals $\mathfrak{M}$ and for all $d \in 2^{\omega}$ (cf. Lemma 0.1).

(b) The analog of the theorem is false in the case of the Baire space $\omega^{\omega}$ since the intersection of all Mycielski ideals is then orthogonal to $\mathbf{K}\left(\omega^{\omega}\right)$ (cf. [R], 4.3).

1.5. Problems. (a) Each Mycielski ideal is orthogonal in fact to the intersection of $\mathbf{K}$ with the ideal $\mathbf{L}$ of Lebesgue negligible sets in $2^{\omega}$ (cf. [My]). Does, for each $A \in \mathbf{L}$, there exist a Mycielski ideal $\mathfrak{M}$ such that $A \in \mathfrak{M}$ ?

(b) If we assume $\mathrm{CH}$, any two Mycielski ideals $\mathfrak{M}, \mathfrak{M}^{\prime}$ are isomorphic (i.e. there is a $1-1$ function $f$ from $2^{\omega}$ onto $2^{\omega}$ such that $\left.A \in \mathfrak{M} \equiv f[A] \in \mathfrak{M}^{\prime}\right)$; cf. $\left[\mathrm{Ba}_{2}\right]$. Is $\mathrm{CH}$ essential? Are any two ideals $\mathfrak{M}$ and $\mathfrak{M}^{\prime}$ Borel isomorphic? 


\section{COMPACT SETS FROM THE IDEAL}

In this section we shall study Mycielski ideals from the point of view of compact sets. Many results in this direction for various ideals can be found in [KLW].

Let $X$ be a perfect Polish space. Let $\mathscr{K}(X)$ denote the space of all compact subsets of $X$, equipped with the Vietoris topology generated by the subbase consisting of the sets $U(G)=\{F \in \mathscr{K}(X): F \subseteq G\}, V(G)=\{F \in \mathscr{K}(X): F \cap$ $G \neq \varnothing\}$ where $G$ denotes an open subset of $X$. It is well known that $\mathscr{K}(X)$ can be metrized by the Hausdorff metric and forms a perfect Polish space which is compact if $X$ is compact (cf. [Ku]).

From now on, let $K$ be a fixed normal set and $\mathfrak{M}$ a fixed Mycielski ideal generated by $\left\{K_{s}\right\}$.

2.1. Proposition. $V_{\mathrm{II}}(K) \cap \mathscr{K}\left(2^{\omega}\right)$ is open in $\mathscr{K}\left(2^{\omega}\right)$.

Proof. Let $F \in V_{\mathrm{II}}(K) \cap \mathscr{K}\left(2^{\omega}\right)$. Thus there is a strategy $\tau$ winning for player II in $\Gamma(F, K)$. Let $D=\left\{d *_{K} \tau: d \in 2^{\omega}\right\}$. Then $D$ is closed. Put $G=2^{\omega} \backslash D$. Thus $G$ is open and $F \in U(G) \subseteq V_{\mathrm{II}}(K) \cap \mathscr{K}\left(2^{\omega}\right)$.

Since $\mathfrak{M}$ contains the family of all finite sets (cf. [My]) forming a dense subset of $\mathscr{K}\left(2^{\omega}\right)$ (cf. [Ku]), we have

2.2. Corollary. $\mathfrak{M} \cap \mathscr{K}\left(2^{\omega}\right) \in \prod_{2}^{0}\left(\mathscr{K}\left(2^{\omega}\right)\right)$. Consequently $\mathfrak{M} \cap \mathscr{K}\left(2^{\omega}\right)$ is residual in $\mathscr{K}\left(2^{\omega}\right)$.

Note that similar properties for Lebesgue negligible sets and meager sets are mentioned in [KLW]; see also [L] where the $\sigma$-porous sets are studied from that standpoint. Following the notation of [KLW], remark that $\mathfrak{M} \cap \mathscr{K}\left(2^{\omega}\right)$ is complete- $\Pi_{2}^{0}$, calibrated and noncontrolled. Moreover, any Borel set which does not belong to $\mathfrak{M}$ contains continuum disjoint compact sets that do not belong to $\mathfrak{M}$. Finally, note that $\mathfrak{M}$ contains "many" compact sets since every perfect set includes a perfect set from $\mathfrak{M}$ (cf. $\left[\mathrm{Ba}_{2}\right]$ ).

2.3. Lemma. If $A \in \mathscr{K}\left(2^{\omega}\right) \cap V_{\mathrm{II}}(K)$ and $\tau$ is a winning strategy for the second player in the game $\Gamma(A, K)$, then there is an integer $N>0$ such that, for each $c \in 2^{\omega}$ with $(\forall k<N, k \in K)(c(k)=\tau(c \mid k))$, we have $c \notin A$.

Proof. Let $A$ and $\tau$ be as in our assumptions. Since $A$ is closed, thus for each $d \in 2^{\omega}$, there is $N>0$ such that $\left[d *_{k} \tau \mid N\right]$ is disjoint from $A$. Let $N(d)$ be the first such $N$. Then the function $d \mapsto N(d)$ is continuous. The compactness of $2^{\omega}$ implies that there is $N_{0}$ such that $\left(\forall d \in 2^{\omega}\right)\left(N(d)<N_{0}\right)$. This $N_{0}$ is good.

2.4. Lemma. For each $A \in \prod_{2}^{0}\left(X \times 2^{\omega}\right)$, the relation $R(x, F) \equiv F \subseteq A_{x}$ is a $\prod_{2}^{0}$ subset of $X \times \mathscr{K}\left(2^{\omega}\right)$.

Proof. It is enough to show that, for an open $G \subseteq X \times 2^{\omega}$, the relation $Q(x, F) \equiv F \subseteq G_{x}$ is open in $X \times \mathscr{K}\left(2^{\omega}\right)$. Indeed, if $Q(x, F)$, then since $F$ is compact, one can find open sets $G_{1} \subseteq X$ and $G_{2} \subseteq 2^{\omega}$ such that $\{x\} \times F \subseteq G_{1} \times G_{2} \subseteq G$. Then $(x, F) \in G_{1} \times U\left(G_{2}\right) \subseteq Q$. 


\section{SECTION PROPERTIES}

For perfect Polish spaces $X, Y$ and a family $\mathfrak{F} \subseteq \mathscr{P}(Y)$, consider a mapping $\Phi_{\mathfrak{F}}: \mathscr{P}(X \times Y) \rightarrow \mathscr{P}(X)$ given by the formula $\Phi_{\mathfrak{F}}(A)=\left\{x \in X: A_{x} \notin \mathfrak{F}\right\}$ for $A \in \mathscr{P}(X \times Y)$. The mapping $\Phi_{\mathfrak{F}}$ is investigated in the literature for the special case where $\mathfrak{F}$ is a $\sigma$-ideal. Two well-known ideals of meager sets and of Lebesgue negligible sets (e.g. in $Y=[0,1]$ ), respectively, are regular in the following sense: if $\mathfrak{J}$ is the $\sigma$-ideal considered, then, for $\alpha<\omega_{1}$, we have $\Phi_{\mathfrak{J}}\left[\sum_{\alpha}^{0}(X \times Y)\right]=\sum_{\alpha}^{0}(X)$ (consequently $\left.\Phi_{\mathfrak{J}}[\operatorname{BOREL}(X \times Y)]=\operatorname{BOREL}(X)\right)$ and $\Phi_{\mathfrak{J}}\left[\sum_{1}^{1}(X \times Y)\right]=\sum_{1}^{1}(X)$. These results can found in [Ke], [V], [Bu]. The equation $\Phi_{\mathfrak{J}}[\operatorname{BOREL}(X \times Y)]=\operatorname{BOREL}(X)$ was applied in [Ma] and [MaSr] to selection problems and in [G] to products of ideals. There are $\sigma$-ideals which are not regular; Shortt in [Sh] gave examples of $\sigma$-ideals $\mathfrak{J}$ satisfying $\Phi_{\mathfrak{J}}[\operatorname{Borel}(X \times Y)]=\sum_{1}^{1}(X)$. Our purpose is to establish images under $\Phi_{\mathfrak{M}}$ of certain Borel and projective pointclasses.

It is easy to prove that, for $A, A_{n} \subseteq X \times 2^{\omega}$, we have

3.1. Lemma. (a) $\Phi_{\mathfrak{M}}(A)=\bigcup\left\{\Phi_{V_{\mathrm{II}\left(K_{s}\right)}}(A): s \in 2^{<\omega}\right\}$,

(b) $\Phi_{\mathfrak{M}}\left(\bigcup\left\{A_{n}: n \in \omega\right\}\right)=\bigcup\left\{\Phi_{\mathfrak{M}}\left(A_{n}\right): n \in \omega\right\}$.

3.2. Proposition. Let $\mathscr{R}$ be a pointclass closed under continuous substitution. Then the following inclusion holds

$$
\left(\exists^{2^{\omega}} \mathscr{R}\right)(X) \cup\left(\forall^{2^{\omega}} \mathscr{R}\right)(X) \subseteq \Phi_{V_{\mathrm{II}}(K)}\left[\mathscr{R}\left(X \times 2^{\omega}\right)\right]
$$

Proof. Let us consider the function $f: 2^{\omega} \rightarrow 2^{\omega}$ from Lemma 0.1. Let $g=$ $(i d, f): X \times 2^{\omega} \rightarrow X \times 2^{\omega}$. Clearly, $g$ is continuous. Now, if $A \in \mathscr{R}\left(X \times 2^{\omega}\right)$, the let $A^{*}=g^{-1}[A]$. Obviously, $A^{*} \in \mathscr{R}\left(X \times 2^{\omega}\right)$. Note that $\Phi_{V_{\mathrm{II}}(K)}\left(A^{*}\right)=$ $\exists^{2^{\omega}} A$ and $\Phi_{V_{\mathrm{II}}(\omega \backslash K)}\left(A^{*}\right)=\forall^{2^{\omega}} A$. Moreover, $\exists^{2^{\omega}} A^{*}=\exists^{2^{\omega}} A$ and $\forall^{2^{\omega}} A^{*}=$ $\forall^{2^{\omega}} A$.

3.3. Proposition. Let $\mathscr{R}$ be a pointclass closed under continuous substitutions. Then $\left(\exists^{2^{\omega}} \mathscr{R}\right)(X) \subseteq \Phi_{\mathfrak{M}}\left[\mathscr{R}\left(X \times 2^{\omega}\right)\right]$ and $\left(\forall^{2^{\omega}} \mathscr{R}\right)(X) \subseteq \Phi_{\mathfrak{M}}\left[\wedge^{\omega} \mathscr{R}\left(X \times 2^{\omega}\right)\right]$.

Proof. Let $A \in \mathscr{R}\left(X \times 2^{\omega}\right)$. The set $A^{*}$ constructed in the previous proof for the sets $A$ and $K_{\langle 0\rangle}$ has the property that $\exists^{2^{\omega}} A=\Phi_{\mathfrak{M}}\left(A^{*}\right)$. Now, let $A_{s}^{*}$ be the set $A^{*}$ constructed in the previous proof for the sets $A$ and $\omega \backslash K_{s}$. Put $A^{* *}=\bigcap\left\{A_{s}^{*}: s \in 2^{<\omega}\right\} \in \wedge^{\omega} \mathscr{R}\left(X \times 2^{\omega}\right)$. Then $x \in \forall^{2^{\omega}} A$ implies $\left(A^{* *}\right)_{x}=$ $\bigcap\left\{\left(A_{s}^{*}\right)_{x}: s \in 2^{<\omega}\right\}=2^{\omega}$, and $x \notin \forall^{2^{\omega}} A$ implies $\left(A^{* *}\right)_{x} \in V_{\mathrm{II}}\left(K_{s}\right)$ for all $s$. Hence $\Phi_{\mathfrak{M}}\left(A^{* *}\right)=\forall^{2^{\omega}} A$.

3.4. Proposition. $\Phi_{\mathfrak{M}}\left[\sum_{1}^{0}\left(X \times 2^{\omega}\right)\right]=\sum_{1}^{0}(X)$.

Proof. For an open $A \subseteq X \times 2^{\omega}$, the set $\Phi_{\mathfrak{M}}(A)$ is equal to the projection of $A$ onto $X$. If $B \subseteq X$ is open, then $B=\Phi_{\mathfrak{M}}\left(B \times 2^{\omega}\right)$. 
3.5. Proposition. $\Phi_{V_{\mathrm{II}}(K)}\left[\Pi_{1}^{0}\left(X \times 2^{\omega}\right)\right] \subseteq \prod_{1}^{0}(X)$.

Proof. Let $A \subseteq X \times 2^{\omega}$ be closed and assume that $x_{0} \notin \Phi_{V_{\mathrm{II}}(K)}(A)$. Then $A_{x_{0}} \in V_{\mathrm{II}}(K)$, so let $\tau$ be a winning strategy for player II in the game $\Gamma(A, K)$. Let $N$ be chosen for $\tau$ by Lemma 2.3. Put $D=\left\{c \in 2^{\omega}:(\forall k<N, k \in\right.$ $K)(c(k)=\tau(c \mid k))\}$. Obviously, $D$ is a clopen subset of $2^{\omega}$, disjoint from $A_{x_{0}}$. For each $c \in D$, we can find open sets $U_{c} \subseteq X$ and $V_{c} \subseteq 2^{\omega}$ such that $x_{0} \in U_{c}, c \in V_{c}$ and $\left(U_{c} \times V_{c}\right) \cap A=\varnothing$. The compactness of $D$ implies that there are $c_{1}, \ldots, c_{n} \in D$ such that $D \subseteq V=V_{c_{1}} \cup \cdots \cup V_{c_{n}}$. Let $U=$ $U_{c_{1}} \cap \cdots \cap U_{c_{n}}$. Then $\left\{x_{0}\right\} \times D \subseteq U \times V$ and $(U \times V) \cap A=\varnothing^{n}$. Moreover, for each $x \in U, \tau$ is a winning strategy for player II in the game $\Gamma\left(A_{x}, K\right)$. Hence $U \cap \Phi_{V_{\mathrm{II}}(K)}(A)=\varnothing$.

From Lemma 3.1(a) we get

\subsection{Corollary. $\Phi_{\mathfrak{m}}\left[\Pi_{1}^{0}\left(X \times 2^{\omega}\right)\right] \subseteq \sum_{2}^{0}(X)$.}

Remark. If $X$ is a $\sigma$-compact space, then the corollary can be proved directly in the following way. Let $A \in \prod_{1}^{0}\left(X \times 2^{\omega}\right)$. Then

$$
x \in \Phi_{\mathfrak{M}}(A) \equiv\left(\exists K \in \mathscr{K}\left(2^{\omega}\right)\right)\left(K \subseteq A_{x} \wedge K \notin \mathfrak{M}\right) \text {. }
$$

The relation $K \subseteq A_{x} \wedge K \notin \mathfrak{M}$ is a $\sum_{2}^{0}$ subset of $X \times \mathscr{K}\left(2^{\omega}\right)$. Since $X \times \mathscr{K}\left(2^{\omega}\right)$ is $\sigma$-compact, the projection of a $\sum_{2}^{0}$ set is a $\sum_{2}^{0}$ set.

3.7. Proposition. $\sum_{2}^{0}(X) \subseteq \Phi_{\mathfrak{m}}\left[\Pi_{1}^{0}\left(X \times 2^{\omega}\right)\right]$.

Proof. Let us assume that $A=\bigcup\left\{A_{n}: n \in \omega\right\}$ where $A_{n}$ 's are closed subsets of $X$. Let $0 \in 2^{\omega}$ be the zero-sequence and $B=(X \times\{0\}) \cup \bigcup\left\{A_{n} \times[0 \mid n]: n \in \omega\right\}$. Then $B$ is a closed subset of $X \times 2^{\omega}$, and $\Phi_{\mathfrak{M}}(B)=A$.

From Lemma 3.1(b) and the above propositions we get

3.8. Theorem. $\Phi_{\mathfrak{M}}\left[\Pi_{1}^{0}\left(X \times 2^{\omega}\right)\right]=\Phi_{\mathfrak{m}}\left[\sum_{2}^{0}\left(X \times 2^{\omega}\right)\right]=\sum_{2}^{0}(X)$.

3.9. Proposition. $\Phi_{\mathfrak{m}}\left[\Pi_{2}^{0}\left(X \times 2^{\omega}\right)\right] \subseteq \sum_{1}^{1}(X)$.

Proof. Let $A \in \prod_{2}^{0}\left(X \times 2^{\omega}\right)$. Note that

$$
A_{x} \notin \mathfrak{M} \equiv\left(\exists F \in \mathscr{K}\left(2^{\omega}\right)\right)\left(F \subseteq A_{x} \wedge F \notin \mathfrak{M}\right) .
$$

The relation $F \subseteq A_{x} \wedge F \notin \mathfrak{M}$ is $\Delta_{3}^{0}$ by Lemma 2.4 and Corollary 2.2. Hence the relation $A_{x} \notin \mathfrak{M}$ is of type $\sum_{1}^{1}$.

\subsection{Theorem.}

(a) $\Phi_{V_{\mathrm{II}}(K)}\left[\prod_{2}^{0}\left(X \times 2^{\omega}\right)\right]=\sum_{1}^{1}(X)$,

(b) $\Phi_{\mathfrak{M}}\left[\Pi_{2}^{0}\left(X \times 2^{\omega}\right)\right]=\Phi_{\mathfrak{m}}\left[\sum_{3}^{0}\left(X \times 2^{\omega}\right)\right]=\sum_{1}^{1}(X)$,

(c) $\prod_{1}^{1}(X) \subseteq \Phi_{V_{\mathrm{II}}(K)}\left[\sum_{2}^{0}\left(X \times 2^{\omega}\right)\right] \subseteq \Delta_{2}^{1}(X)$,

(d) $\prod_{1}^{1}(X) \cup \sum_{1}^{1}(X) \subseteq \Phi_{\mathfrak{m}}\left[\prod_{3}^{0}\left(X \times 2^{\omega}\right)\right] \subseteq \Phi_{\mathfrak{m}}\left[\operatorname{BOREL}\left(X \times 2^{\omega}\right)\right] \subseteq \Delta_{2}^{1}(X)$. 
Proof. For (a), use Proposition 3.2 and a slight modification of Proposition 3.9. For (b), use Propositions 3.3, 3.9 and Lemma 3.1. The first inclusion of (c) is a consequence of Proposition 3.2. The second inclusion is a consequence of Borel determinacy (cf. [Mar]): for a Borel set $A \subseteq X \times 2^{\omega}$, we have $A_{x} \notin$ $V_{\mathrm{II}}(K) \equiv(\forall \tau)(\exists \sigma)\left(\left(x, \sigma *_{K} \tau\right) \in A\right) \equiv(\exists \sigma)(\forall \tau)\left(\left(x, \sigma{ }{ }_{K} \tau\right) \in A\right)$. In the first inclusion of (d) use (b) and Proposition 3.3, in the last use the proof of (c).

3.11. Theorem.

(a) $\Phi_{V_{\mathrm{II}}(K)}\left[\sum_{n}^{1}\left(X \times 2^{\omega}\right)\right]=\prod_{n+1}^{1}(X)$,

(b) $\Phi_{\mathfrak{m}}\left[\sum_{n}^{1}\left(X \times 2^{\omega}\right]=\prod_{n+1}^{1}(X)\right.$,

(c) $\left.\sum_{n+1}^{1}(X) \subseteq \Phi_{V_{\mathrm{II}}(K)} \prod_{n}^{1}\left(X \times 2^{\omega}\right)\right] \subseteq \prod_{n+2}^{1}(X)$,

(d) $\sum_{n+1}^{1}(X) \subseteq \Phi_{\mathfrak{m}}\left[\prod_{n}^{1}\left(X \times 2^{\omega}\right)\right] \subseteq \prod_{n+2}^{1}(X)$.

Under $\prod_{n}^{1}$ determinacy we can get $\sum_{n+1}^{1}(X)$ on the right sides of $(\mathrm{c})$ and $(\mathrm{d})$.

Proof. Propositions 3.2 and 3.3 give that

$$
\begin{gathered}
\prod_{n+1}^{1}(X) \subseteq \Phi_{V_{\mathrm{II}}(K)}\left[\sum_{n}^{1}\left(X \times 2^{\omega}\right)\right] \cap \Phi_{\mathfrak{M}}\left[\sum_{n}^{1}\left(X \times 2^{\omega}\right)\right] \text { and } \\
\sum_{n+1}^{1}(X) \subseteq \Phi_{V_{\mathrm{II}}(K)}\left[\prod_{n}^{1}\left(X \times 2^{\omega}\right)\right] \cap \Phi_{\mathfrak{M}}\left[\prod_{n}^{1}\left(X \times 2^{\omega}\right)\right] .
\end{gathered}
$$

For the remaining inclusions, note that if $A \in \sum_{n}^{1}\left(X \times 2^{\omega}\right)$, then $A_{x} \notin V_{\mathrm{II}}(K) \equiv$ $(\forall \tau)(\exists \sigma)\left(\left(x, \sigma *_{K} \tau\right) \in A\right)$. The right-side formula presents a $\prod_{n+1}^{1}$ subset of $X$. Hence $\Phi_{\mathfrak{M}}(A)$ and $\Phi_{V_{\mathrm{II}}(K)}(A)$ are $\prod_{n+1}^{1}$. Similarly, if $A \in \prod_{n}^{1}\left(X \times 2^{\omega}\right)$ then the relations $A_{x} \notin \mathfrak{M}$ and $A_{x} \notin V_{\mathrm{II}}(K)$ are of type $\prod_{n+2}^{1}$. If we assume $\prod_{n}^{1}$ determinacy, these relations become of type $\sum_{n+1}^{1}$ by changing the places of the quantifiers.

3.12. Problem. Establish $\Phi_{V_{\mathrm{II}}(K)}\left[\operatorname{BOREL}\left(X \times 2^{\omega}\right)\right]$ and $\Phi_{\mathfrak{m}}\left[\operatorname{BOREL}\left(X \times 2^{\omega}\right)\right]$.

Finally, let us observe that $\mathfrak{M}$ has not the Fubini property.

3.13. Proposition. There exists a set $A \in \prod_{2}^{0}\left(2^{\omega} \times 2^{\omega}\right)$ such that all vertical sections $A_{c}$ do not belong to $\mathfrak{M}$, while all horizontal sections $A^{d}$ constitute a basis of $\mathfrak{M}$.

Proof. Let $\pi: 2^{<\omega} \rightarrow \omega$ be a bijection, and let $\left\{L_{i}: i \in \omega\right\}$ be a family of disjoint infinite subsets of $K_{\langle 0\rangle}$. For $d \in 2^{\omega}, i \in \omega$ and $s \in 2^{<\omega}$, put $(d)_{i}(s)=$ $d\left(l_{\pi(s)}^{i}\right)$ where $L_{i}=\left\{l_{c}^{i}, l_{1}^{i}, \ldots\right\}$. Let us define $(c, d) \in A \equiv(\forall s)(\exists j)(j \in$ $\left.K_{s} \wedge(d)_{\pi(s)}(c \mid j) \neq c(j)\right)$. Clearly, $A \in \prod_{2}^{0}\left(2^{\omega} \times 2^{\omega}\right)$ and the horizontal sections $A^{d}$ form a basis. Moreover, $A_{c}$ 's do not belong to $\mathfrak{M}$ since player II has no winning strategy in $\Gamma\left(A_{c}, K_{\langle 1\rangle}\right)$. 


\section{REFERENCES}

[ $\left.\mathrm{Ba}_{1}\right]$ M. Balcerzak, The decomposition property of $\sigma$-ideals II, Rad. Mat. 3 (1987), 261-266.

$\left[\mathrm{Ba}_{2}\right] \_$, On $\sigma$-ideals having perfect members in all perfect sets, preprint.

[Bu] J. P. Burgess, Classical hierarchies from a modern standpoint. Part I. C-sets, Fund. Math. 115 (1983), 81-95.

[G] M. Gavalec, Iterated products of ideals of Borel sets, Colloq. Math. 50 (1985), 39-52.

[Ke] A. S. Kechris, Measure and category in effective descriptive set theory, Ann. Math. Logic 5 (1973), 337-384.

[KLW] A. S. Kechris, A. Louveau and W. H. Woodin, The structure of $\sigma$-ideals of compact sets, Trans. Amer. Math. Soc. 301 (1987), 263-288.

[Ku] K. Kuratowski, Topology, vols. I and II, Academic Press, New York, 1966, 1968.

[L] L. Larson, Typical compact sets in the Hausdorff metric are porous, Real Anal. Exchange 13 (1987-88), 116-118.

[Ma] A. Maitra, Selectors for Borel sets with large sections, Proc. Amer. Math. Soc. 89 (1983), 705-708.

[MaSr] A. Maitra and V. V. Srivatsa, Parametrizations of Borel sets with large sections, Proc. Amer. Math. Soc. 93 (1985), 545-548.

[Mar] D. A. Martin, Borel determinacy, Ann. of Math. 102 (1975), 363-371.

[Mo] Y. N. Moschovakis, Descriptive set theory, North-Holland, Amsterdam, 1980.

[My] J. Mycielski, Some new ideals of sets on the real line, Colloq. Math. 20 (1969), 71-76.

[R] A. Rosłanowski, On game ideals, preprint.

[Sh] R. M. Shortt, Product sigma-ideals, Topology Appl. 23 (1986), 279-290.

[V] R. L. Vaught, Invariant sets in topology and logic, Fund. Math. 82 (1974), 269-294.

Institute of Mathematics, Lódż University, Ul.S.Banacha 22, 90-238 Lódż, Poland

Mathematical Institute, University of Wroclaw, Pl.GrunWaldzki 2/4, 50-384 Wroclaw, Poland 